\title{
Impact of Costco Brand Name Hearing Aid Expansion on Otolaryngology Office Hearing Aid Revenue
}

\author{
Adam E Block* \\ Department of Public Health, New York Medical College School of Health Sciences and \\ Practice, New York, USA
}

*Corresponding author: Adam E Block, Department of Public Health, New York Medical College School of Health Sciences and Practice 40 Sunshine Cottage Road, Skyline Building, NY10595, New York, USA, Fax: 914-594-2049; Tel: 914-594-2041/ 917-209-6553; Email: ablock4@nymc.edu

\section{Short Communication}

Volume 4 Issue 1

Received Date: June 20, 2019

Published Date: June 26, 2019

DOI: $10.23880 /$ ooaj-16000180

\section{Abstract}

Objectives: The objective of this study is to evaluate the impact of Costco's expansion in the hearing aid market on the impact of hearing aid sales by otolaryngologists.

Design: Revenue data was acquired for 34 practice locations for the years 2013 through 2017 and compared before and after 2015, when Costco expanded its options for hearing aid brands. Revenue growth was regressed on the distance between otolaryngology office and the nearest three Costco locations.

Results: A univariate regression analysis shows that a 10-minute decrease in average travel time to Costco would result in an $8 \%$ increase in CAGR growth.

Conclusion: This study shows the closer an otolaryngologist's office is to a Costco, the greater the growth the office experienced after 2015, indicating that Costco proximity did not reduce hearing aid sales.

Abbreviations: CAGR: Compound Annual Growth Rate.

\section{Introduction}

Hearing loss is prevalent, but vastly undertreated in the United States. In the United States 26.7M people over the age of 50 have at least mild hearing loss, however, over $3.8 \mathrm{M}$ or $14 \%$ utilize a hearing aid [1]. Hearing aids are not covered by traditional Medicare, the predominant source of coverage for adults in the United States over age 65 [2]. This gap between hearing loss prevalence and treatment rate suggests that cost could be one driver of the under-treatment of hearing loss with hearing aids [3]. Therefore, the cost is predominantly out of pocket for this population.
Generally, diagnostic hearing tests are first administered, hearing is evaluated and any recommended options for hearing correction are provided to the patient who can then elect to have the hearing correction tailored and then evaluated by a clinical team including otolaryngologists, audiologists and hearing aid specialists. Costco is a membership-only wholesaler with 531 locations in the United States that offers large volumes of products at discounted prices. It also offers Costco Optical which will provide an optometry visit and heavily discounted corrective lenses and is the fifth largest optical retailer in the United States [4]. Recently, Costco has broadened its hearing aid business providing a lower cost alternative for a broader set of brand name hearing aids. Costco, which had once offered only lower level options including Kirkland, Bernafon and older Resound technology began to offer non-differentiated higher end 


\section{Otolaryngology Open Access Journal}

brands including Phonak in 2015, creating greater competition with otolaryngologists [5]. The typical health economic implication is that the expansion of lower cost alternatives will negatively impact the market for the remaining competitors and a recent study by Audigy provides preliminary evidence of the competition effect [6]. However, there is an alternative possibility and this study provides preliminary evidence that Costco's marketing reach on the value of hearing aids and their underutilization broadens the entire market's demand for hearing aids resulting in growth of the entire market. This paper tests the possibility that Costco's growth in the hearing aid market has yielded growth for the clinical practices offering hearing aids to patients.

\section{Materials and Methods}

To analyze the impact of Costco expansion, proprietary data was aggregated 34 otolaryngology locations. Data includes revenue information from years 2013 through 2017 for all hearing aid purchases by site of purchase. Because of the change in Costco's offerings before and after January 1, 2015, the pre-period was defined as calendar years 2013 and 2014 and the post period is years 2015, 2016 and 2017. Annual revenue growth by site was compiled using compound annual growth rate (CAGR). The impact of the 2015 change on each site is the difference between the 2015-2017 CAGR and the 2013-2014 CAGR. Any site not in existence in all five years was excluded from the study.

The estimated driving time between each office location and the three closest Costco locations to each otolaryngology location was calculated. The Costco location drive times were developed using driving time estimates from Tableau Version 10.5. To capture the variation in Costco's marketing reach on each office's target market we use the average travel time between the otolaryngology office and the three closest Costco locations. Travel times are used because the region encompasses both urban and rural settings and travel time for 15-miles in an urban setting is far longer than travel time for 15 miles in a rural setting. The shorter the travel time, the greater the impact of Costco's marketing has on the hearing aid market and therefore, the greater the likelihood that patients are making a choice between Costco and a clinical practice for hearing correction. This study compares the results from 34 practice locations with complete revenue data over the entire period. Drive times were capped at 60 minutes because we think it is unlikely that people plan to travel in excess of 60 minutes to a Costco in the region of study. To test the hypothesis that lower drive times to Costco positively impacts hearing aid revenue growth, we used a descriptive analysis and a univariate regression analysis.

\section{Results}

The data for the 34 locations over five years compared to minimum drive times between the offices provides some directional results. The means of the CAGR difference is $-13 \%$ and the means of the three shortest drive times between the practice location and Costco locations is 29.4 minutes.

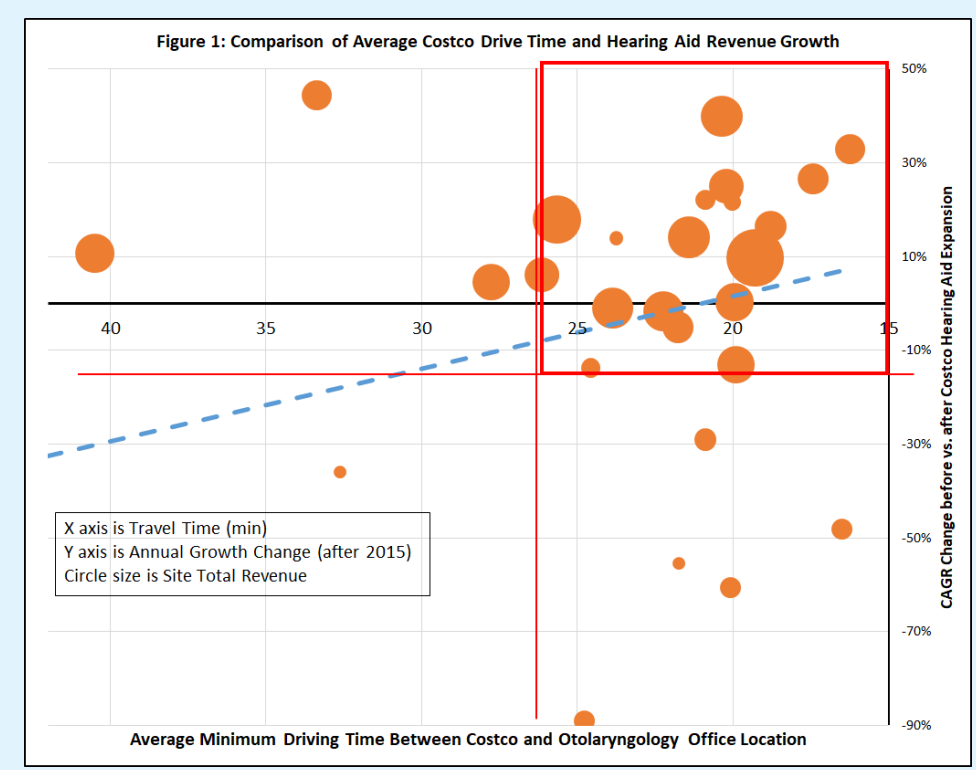

Figure 1: Comparison of Average Costco Drive Time and Hearing Aid Revenue Growth. 


\section{Otolaryngology Open Access Journal}

Figure 1 show the visualization of the quadrant analysis where the upper right quadrant represents locations with above average revenue growth but below average drive times to Costco. Quadrant 1 of Figure 1 is the upper right hand boxed portion and means that when drive times are shorter, growth is higher at the location. Table 1 shows that of the 34 locations, 56\% were in Quadrant 1 . When the locations are weighted by their total five-year revenue, $65 \%$ of the revenue weighted locations were in Quadrant 1.

\begin{tabular}{|c|c|c|c|}
\hline Quadrant & Count & Percent Count & Revenue Weighted Quadrant \\
\hline $\mathbf{1}$ & $\mathbf{1 9}$ & $\mathbf{5 6 \%}$ & $\mathbf{6 5 \%}$ \\
\hline 2 & 6 & $18 \%$ & $20 \%$ \\
\hline 3 & 3 & $9 \%$ & $9 \%$ \\
\hline 4 & 6 & $18 \%$ & $6 \%$ \\
\hline Total & 34 & $100 \%$ & $100 \%$ \\
\hline
\end{tabular}

Table 1: Otolaryngology Practice Locations by Quadrant.

Lastly, a simple univariate OLS regression estimating the impact of average travel time between Costco and otolaryngology practice locations found that a 10-minute decrease in average travel time would result in an $8 \%$ increase in CAGR growth between the before period (2013-2014) and the after period (2015-2017). This suggests that closer presence of Costco actually increases the hearing aid revenue growth at these locations.

\section{Discussion}

The research provides preliminary evidence that the inception of big box retailers such as Costco into the audiology space may grow the market size and not simply cannibalize market share from existing competitors, particularly clinical practices. This evidence uses data from a single multispecialty practice to counter evidence from an Audigy study [6] that proximity to Costco reduces hearing aid sales among otolaryngology practices. This is a complex issue and evidence will vary based on the market: while the hearing aid market may see growth among all competitors, this market is distinct from others because hearing correction is grossly underutilized.

\section{References}

1. Peracino A (2015) Hearing loss and dementia in the aging population. Audiol Neurotol 19(1): 6-9.

2. Medicare Consumer Guide. What to do if you're on medicare and need a hearing aid.

3. Manchaiah V, Taylor B, Dockens AL, Tran NR, Lane K, et al. (2017) Applications of direct-to-consumer hearing devices for adults with hearing loss: A review. Clin Interv Aging 12: 859-871.

4. Staff (2016) Snapshots of optical's 10 largest US retail players.

5. Kirkwood D (2014) Phonak confirms that it will distribute hearing aids through costco.

6. Barrett LL, Connon JS (2017) The costco effect. The Hearing Review. 